\title{
Instrumentos de avaliação utilizados por terapeutas ocupacionais na criança com paralisia braquial obstétrica
}

\author{
Thaianny Taís Dantas de Brito, Carolinne Linhares Pinheiro
}

Instituto Federal de Educação, Ciência e Tecnologia do Rio de Janeiro - IFRJ, Rio de Janeiro, RJ, Brasil.

\begin{abstract}
Resumo: Introdução: A paralisia braquial obstétrica (PBO) é resultado de lesão do plexo braquial ao nascimento, podendo ocasionar disfunções do membro superior acometido, repercutindo de forma significativa na vida da criança. Ao avaliar a criança com PBO, o terapeuta ocupacional pode fazer uso de instrumentos de avaliação, devendo ter conhecimentos para escolher e aplicar o instrumento mais apropriado. Objetivo: O objetivo desta revisão foi analisar a literatura sobre utilização de instrumentos de avaliação por terapeutas ocupacionais na criança com PBO. Método: A busca dos artigos foi realizada nas bases de dados Scirus, Cinahl, Medline, Psycinfo, Scopus e Lilacs, atendendo aos critérios de seleção: estudos realizados em crianças com PBO de 0 a 12 anos, nos idiomas inglês, português e espanhol, publicados nos últimos 10 anos, que tivessem o terapeuta ocupacional como um dos autores e/ou reportassem a aplicação do instrumento por este profissional. Resultados: Foram encontrados 15 estudos, provenientes de seis países que reportaram 17 instrumentos de avaliação, dos quais cinco desenvolvidos recentemente eram específicos para essa clientela. Não foi identificada a necessidade de modificar os instrumentos não específicos para a aplicação nas crianças com PBO, demonstrando que estes podem ser usados em seu formato original, facilitando o uso na prática clínica. A maioria dos instrumentos contemplou aspectos relacionados ao desempenho ocupacional inseridos no domínio de Atividade e Participação da Classificação Internacional de Funcionalidade, indicando a preocupação deste profissional em adequar o processo de avaliação da criança com PBO ao paradigma atual de compreensão de saúde e ao campo da Terapia Ocupacional.
\end{abstract}

Palavras-chave: Paralisia Braquial Obstétrica, Criança, Avaliação, Terapia Ocupacional.

\section{Assessment tools used by occupational therapists in children with obstetric brachial plexus palsy}

\begin{abstract}
Introduction: The Obstetric Brachial Plexus Palsy (OBPP) is a result of brachial plexus injury at birth and may cause dysfunction of the affected upper limb, reflecting significantly in the child's life. When evaluating a child with OBPP the occupational therapist can use evaluation tools, and has to have knowledge to choose and apply the most appropriate instrument. Objective: This review aimed to analyze the literature on the use of evaluation tools by occupational therapists in children with OBPP. Method: The search for articles was performed in the databases Scirus, Cinahl, Medline, Psycinfo, Scopus and Lilacs using the following selection criteria: studies with 0-12 years old children with OBPP in English, Portuguese and Spanish, published in the last 10 years, with the occupational therapist as one of the authors and/or reporting the application of the instrument by this professional. Results: There were 15 studies from six countries that reported 17 evaluation instruments, five of which, recently developed, were specific to this clientele. The study did not identify the need to modify the non-specific instruments to the application in children with OBPP, demonstrating that they can be used in its original format, facilitating the use in the clinical practice. Most instruments included aspects related to occupational performance inserted in the field of Activity and Participation of the International Classification of Functioning, indicating the concern of this professional in suiting the assessment process of the child with the OBPP to the current paradigm of health understanding and occupational therapy field.
\end{abstract}

Keywords: Obstetric Brachial Plexus Palsy, Child, Assessment, Occupational Therapy.

Autor para correspondência: Carolinne Linhares Pinheiro, Coordenação de Terapia Ocupacional, Instituto Federal de Educação, Ciência e Tecnologia do Rio de Janeiro, Campus Realengo, Rua Professor Carlos Wenceslau, 343, Realengo, CEP 21715-000, Rio de Janeiro, RJ, Brasil, e-mail: carolinne.pinheiro@ifrj.edu.br.

Recebido em Fev. 14, 2014; $1^{\text {a }}$ Revisão em Nov. 21, 2014; $2^{\text {a }}$ Revisão em Ago. 3, 2015; Aceito em Out. $17,2015$. 


\section{Introdução}

A paralisia braquial obstétrica $(\mathrm{PBO})$ é resultado de uma lesão do plexo braquial ao nascimento, podendo se tratar de uma lesão parcial ou total da rede de nervos desse plexo (HO; CURTIS; CLARKE, 2012; VAZ et al., 2010). Essa rede é responsável pela inervação dos membros superiores, sendo constituída pelas raízes cervicais $\mathrm{C} 5, \mathrm{C} 6, \mathrm{C} 7$ e C8, além da raiz torácica $\mathrm{T} 1$, e o estiramento, compressão ou avulsão radicular dos troncos nervosos pode provocar a lesão (GHIZONI et al., 2010; ZAFEIRIOU; PSYCHOGIOU, 2008).

A incidência de PBO, de acordo com Hoeksma, Wolf e Oei (2000), varia de 0,9 a 2,3 por 1.000 nascidos vivos. Alguns fatores de risco para a paralisia braquial obstétrica incluem: macrossomia fetal, diabetes, baixa estatura da mãe, trabalho de parto demorado, apresentação pélvica, aumento de peso acima de $12 \mathrm{~kg}$ na gravidez, asfixia perinatal, utilização de fórceps (GHIZONI et al., 2010; ZAFEIRIOU; PSYCHOGIOU, 2008). Ainda em consonância com Ghizoni et al. (2010), as lesóes em neonatos sem causa obstétrica sugerem associação com outras etiologias, como a má-formação do plexo braquial ou superdistensão devido à atitude fetal intraútero.

A severidade do acometimento funcional e o prognóstico da $\mathrm{PBO}$ dependem do tipo da lesão, que variam desde neuropraxia, que é a lesão leve com perda motora e sensitiva, sem alteração estrutural, até a neurotmese, que é a ruptura completa do nervo (BIALOCERKOWSKI; GELDING, 2006; PALMGREN et al., 2007; SIQUEIRA, 2007). A severidade do acometimento também depende da extensão da lesão nas estruturas do plexo, podendo envolver desde um nervo até todas as raízes nervosas (BIALOCERKOWSKI; GELDING, 2006; PALMGREN et al., 2007).

$\mathrm{O}$ parâmetro de classificação clínica da $\mathrm{PBO}$ é estabelecido de acordo com as raízes nervosas envolvidas, cujas lesôes que afetam o tronco superior do plexo (C5 - C6, às vezes $\mathrm{C} 7)$ são chamadas de paralisia alta ou de Erb-Duchenne; as que afetam o tronco inferior (C8 - T1) são chamadas de paralisia baixa ou de Klumpke; e ainda há a paralisia completa, na qual todas as raízes nervosas estão envolvidas (GHIZONI et al., 2010; ZAFEIRIOU; PSYCHOGIOU, 2008).

Na paralisa de Erb-Duchenne, que corresponde à maioria dos casos de $\mathrm{PBO}$ e é o tipo que apresenta melhor prognóstico, o recém-nascido apresenta paralisia dos músculos abdutores e rotadores externos do ombro, o padrão do membro é ombro aduzido e em rotação interna, cotovelo em extensão, antebraço em pronação, punho em flexão e dedos estendidos. Já na paralisia de Klumpke, o padrão do membro é de flexão do cotovelo, supinação do antebraço e paralisia da mão com ausência do reflexo de preensão palmar, enquanto os músculos mais proximais estão intactos. Na paralisia completa, o membro superior apresenta paralisia flácida, com mão em garra e alteração da sensibilidade (SILVA et al., 2010; ZAFEIRIOU; PSYCHOGIOU, 2008).

Desse modo, a paralisia braquial obstétrica leva frequentemente a disfunçóes do membro acometido, que podem acarretar em prejuízos nas funçôes sensoriais e motoras da criança, incluindo perda de amplitude de movimento ativo, déficit de força muscular, deformidades e/ou contraturas articulares e déficits sensitivos (HO; CURTIS; CLARKE, 2012).

Para além dos problemas neuromusculoesqueléticos, a $\mathrm{PBO}$ pode acarretar implicaçôes no desenvolvimento neuropsicomotor das crianças, que geralmente apresentam atrasos no desenvolvimento da coordenação visomotora, dificuldade na manipulação de objetos, no desempenho de tarefas bimanuais, no alcance de marcos motores como sentar, rolar e engatinhar (SPAARGAREN et al., 2011). Dessa forma, a PBO pode ter repercussão significativa na vida da criança e resultar em limitaçóes no desempenho das atividades da vida diária e na participaçáo da criança na escola e na comunidade (VAZ et al., 2010). Diante das alteraçóes estruturais, funcionais, emocionais e do comprometimento no desempenho ocupacional, o terapeuta ocupacional é um dos profissionais que pode compor a equipe para o tratamento de crianças com PBO (GHIZONI et al., 2010; HO; CURTIS; CLARKE, 2012; SPAARGAREN et al., 2011).

Segundo a American Occupational Therapy Association (BRAYMAN et al., 2005), a avaliação é definida como o processo de obtenção e interpretação dos dados necessários para a intervenção, incluindo a utilização de ferramentas ou instrumentos específicos, o planejamento e a documentação desse processo e dos seus resultados. A avaliação auxilia no planejamento e estabelecimento das metas e objetivos do tratamento, bem como guia a intervenção e evidencia os resultados obtidos (CHAVES et al., 2010; HO; CURTIS; CLARKE, 2012).

Cada vez mais é necessário o embasamento científico nas intervençóes da Terapia Ocupacional e a comprovação dos seus resultados por meio de avaliaçôes padronizadas. Tal embasamento serve não somente para favorecer a eficácia da intervenção, como também para ser um aspecto norteador no momento da escolha e planejamento do tratamento (CHAVES et al., 2010). As avaliações padronizadas 
também favorecem o reconhecimento clínico e científico da Terapia Ocupacional, viabilizam a produção de conhecimento específico e conferem confiabilidade às intervençôes (CHAVES et al., 2010).

Instrumentos de avaliação padronizados têm como característica possuírem procedimento padrão para sua administração e pontuação, informaçóes sobre sua confiabilidade e validade, que são fundamentais para a correta interpretação dos resultados (UNSWORTH, 2000).

É importante que os terapeutas ocupacionais tenham conhecimento dos recursos disponíveis, dentro e fora do país, para escolher e aplicar corretamente o instrumento mais apropriado para a clientela em questão (ROCHA; DORNELAS; MAGALHÂES, 2013).

Assim sendo, esse estudo teve como objetivo analisar a produção da literatura sobre os instrumentos de avaliação utilizados por terapeutas ocupacionais na criança com PBO, buscando apresentar e caracterizar os instrumentos usados nesta clientela, identificando o objetivo destes, assim como a faixa etária que foi utilizado, e apontar se houve necessidade de modificação da avaliação para utilização em crianças com PBO.

\section{Método}

Esse é um estudo de revisão de literatura, e a busca dos artigos ocorreu no período de julho a dezembro de 2012, nas bases de dados SCIRUS, CINAHL, Medline via PubMed, PsycINFO, SCOPUS e LILACS. Os descritores utilizados para a busca foram: occupational therapy, child, evaluation e brachial plexus neuropathies; e os termos de busca utilizados incluíram assessment, brachial plexus injury, obstetric brachial palsy, occupational therapist, brachial plexus birth injuries e brachial plexus birth palsy. Para a busca nas bases de dados, os termos foram utilizados em pares, combinadas entre si.

Occupational Therapy $>$ Brachial plexus neuropathies

Occupational Therapy $>$ Brachial plexus injury

Occupational Therapy > Obstetric brachial palsy

Occupational Therapy > Brachial plexus birth injuries

Occupational Therapy > Brachial plexus birth palsy

Occupational Therapist $>$ Brachial plexus neuropathies
Occupational Therapist> Brachial plexus injury

Occupational Therapist > Obstetric brachial palsy

Occupational Therapist $>$ Brachial plexus birth injuries

Occupational Therapist $>$ Brachial plexus birth palsy

Assessment $>$ Brachial plexus neuropathies

Assessment > Brachial plexus injury

Assessment > Obstetric brachial palsy

Assessment $>$ Brachial plexus birth injuries

Assessment > Brachial plexus birth palsy

Evaluation > Brachial plexus neuropathies

Evaluation > Brachial plexus injury

Evaluation > Obstetric brachial palsy

Evaluation > Brachial plexus birth injuries

Evaluation > Brachial plexus birth palsy.

Brachial plexus neuropathies $>$ Child

Os critérios de seleção dos artigos definidos foram: estudos realizados em crianças com $\mathrm{PBO}$ na faixa etária de 0 a 12 anos, nos idiomas inglês, português e espanhol, publicados nos últimos 10 anos (2003 a 2012), apontando instrumentos de avaliação utilizados por terapeutas ocupacionais e/ou estudos com pelo menos um dos autores com formação na área de Terapia Ocupacional.

Sendo assim, primeiramente foi feita a análise dos títulos, excluindo artigos que informavam já no título que não se adequavam aos critérios de seleção, como, por exemplo, estudos somente de adultos com lesão do plexo braquial. Por meio dessa primeira análise, foram selecionados 368 artigos; posteriormente, seguiu-se a análise dos resumos, e o número de artigos foi reduzido para 191. Eliminando as repetiçôes, restaram 36 artigos.

Foi realizada a leitura na íntegra dos 36 estudos, com o propósito de analisar a consonância com aos critérios de seleção descritos. Após essa etapa, o número final foi de 15 artigos incluídos nesta revisão.

\section{Resultados}

Foram encontrados 15 estudos, 14 em inglês e 1 em português, que reportaram instrumentos utilizados por terapeutas ocupacionais para a 
avaliação de crianças com PBO. Dentre os estudos selecionados, apontados na Tabela 1, 10 tiveram como autor principal um terapeuta ocupacional, em três o autor principal era médico, e nos dois restantes, fisioterapeuta. As publicaçóes foram provenientes de seis países diferentes, sendo a Suécia o país com maior número de publicaçôes $(\mathrm{n}=4)$, em seguida a Holanda e os Estados Unidos ( $\mathrm{n}=3$ ), seguidos do Brasil e do Canadá ( $n=2)$, e da Finlândia $(n=1)$.

A faixa etária predominante da populaçáo envolvida nos estudos foi de 4 a 7 anos. A amostra em cinco dos estudos era mista, envolvendo crianças com outros acometimentos, e em dez a amostra era somente de crianças com PBO.

No que se refere aos objetivos dos estudos incluídos nessa revisão, dois deles avaliaram questóes referentes à estrutura e funçóes do corpo de crianças com $\mathrm{PBO}$ (IMMERMAN et al., 2011; PALMGREN et al., 2007). Em sete estudos, o objetivo relacionava-se a investigar avaliaçôes que se adequassem a essa população (BOESCHOTEN et al., 2007; HO; CURTIS; CLARKE, 2006, 2012; KRUMLINDESUNDHOLM; ELIASSON, 2003; MOURA; MAKITA; OLIVEIRA, 2004; MULCAHEY et al., 2012; SKOLD et al., 2011), e quatro destes reportaram o processo de desenvolvimento de alguns instrumentos específicos para essa clientela, como o Assisting Hand Assessment (AHA), Children's Hand-use Experience Questionnaire (CHEQ), Brachial Plexus Outcome Measure (BPOM) e Set of Activities. Os estudos que descreveram o desenvolvimento de instrumentos de avaliação também apontaram e mensuraram suas propriedades psicométricas.

Três pesquisas tiveram como objetivo principal demonstrar e avaliar as propriedades psicométricas de instrumentos como a AHA e a Medida Canadense do Desempenho Ocupacional (HOLMEFUR; KRUMLINDE-SUNDHOM; ELIASSON, 2007; KRUMLINDE-SUNDHOLM et al., 2007; VERKERK et al., 2006). Dois artigos tinham como intuito documentar resultado de intervenção cirúrgica e de reabilitação nesta população, utilizando os seguintes instrumentos: Toddler Arm Use Test $\mathrm{e}$ o Pediatric Outcomes Data Collection Instrument (DEDINI et al., 2008; VAZ et al., 2010). E, por fim, um estudo teve por objetivo avaliar o desempenho ocupacional da criança com $\mathrm{PBO}$ (SPAARGAREN et al., 2011).

Foram reportados 17 instrumentos de avaliaçáo que estão identificados na Tabela 2, dos quais somente cinco - Activitie Movement Scale (AMS), Set of Activities, Children's Hand-use Experience Questionnaire (CHEQ), The Brachial Plexus Outcome Measure (BPOM), Assisting Hand
Assessment (AHA) - foram desenvolvidos para avaliar especificamente crianças com paralisia braquial obstétrica.

Os demais instrumentos de avaliação (12), a saber - Vineland Adaptive Behavior, ABILHAND-kids, Children's Assessment of Participation and Enjoyment (CAPE), Monofilamentos de Semmes-Weisntein, Toddler Arm Use Test (TAUT), Inventário de Avaliação Pediátrica de Incapacidade (PEDI), Nine-hole peg test (9-HPT), Box and Block Test (BBT), Teste de estereognosia, Pediatric Outcomes Data Collection Instrument (PODCI), Medida Canadense do Desempenho Ocupacional (COPM), e o TNO AZL Preschool Quality of Life (TAPQOL) ou TNO AZL Children's Quality of Life (TACQOL) -, foram desenvolvidos para outras populaçôes e utilizados nos estudos com crianças com PBO.

Alguns estudos analisados nesta revisão discorreram sobre a aplicabilidade de alguns desses instrumentos não específicos para crianças com $\mathrm{PBO}$.

O 9-HPT é simples e comercialmente disponível, e Immerman et al. (2011) apontam que uma possível limitação do instrumento ao ser utilizado em crianças com $\mathrm{PBO}$ é que ele requer função do ombro ativo, a fim de posicionar a máo para realizar o teste, e um déficit residual do ombro, frequente na $\mathrm{PBO}$, poderia afetar os resultados.

Os resultados do estudo de Mulcahey et al. (2012) sobre a utilização do BBT em crianças com $\mathrm{PBO}$ mostraram que o instrumento tem a capacidade para prever o nível de prejuízo nessas crianças. É um instrumento de baixo custo, recomendado para avaliar a função da máo de crianças com PBO, porém não se mostrou um instrumento significativo para a avaliação da função do ombro. Ainda nesse estudo, o Teste de Estereognosia se mostrou capaz de prever o grau de comprometimento das crianças com PBO.

Os monofilamentos de Semmes Weinstein é um instrumento padronizado que pode ser usado com segurança em crianças, cujo registro é obtido de forma simples e sem dor (PALMGREN et al., 2007).

Ho, Curtis e Clarke (2006) revelaram que o PEDI não é capaz de discriminar a capacidade de autocuidado entre crianças com $\mathrm{PBO}$ e seus pares. Porém, ele foi capaz de distinguir o desempenho de crianças com diferentes gravidades de $\mathrm{PBO}$.

No estudo de Verkerk et al. (2006), a amostra náo foi escolhida de acordo com diagnóstico, pois a COPM não está diretamente ligada a um diagnóstico. O estudo sugeriu que os problemas identificados pela COPM são consistentes o suficiente para identificação de metas para a Terapia Ocupacional e reabilitação 


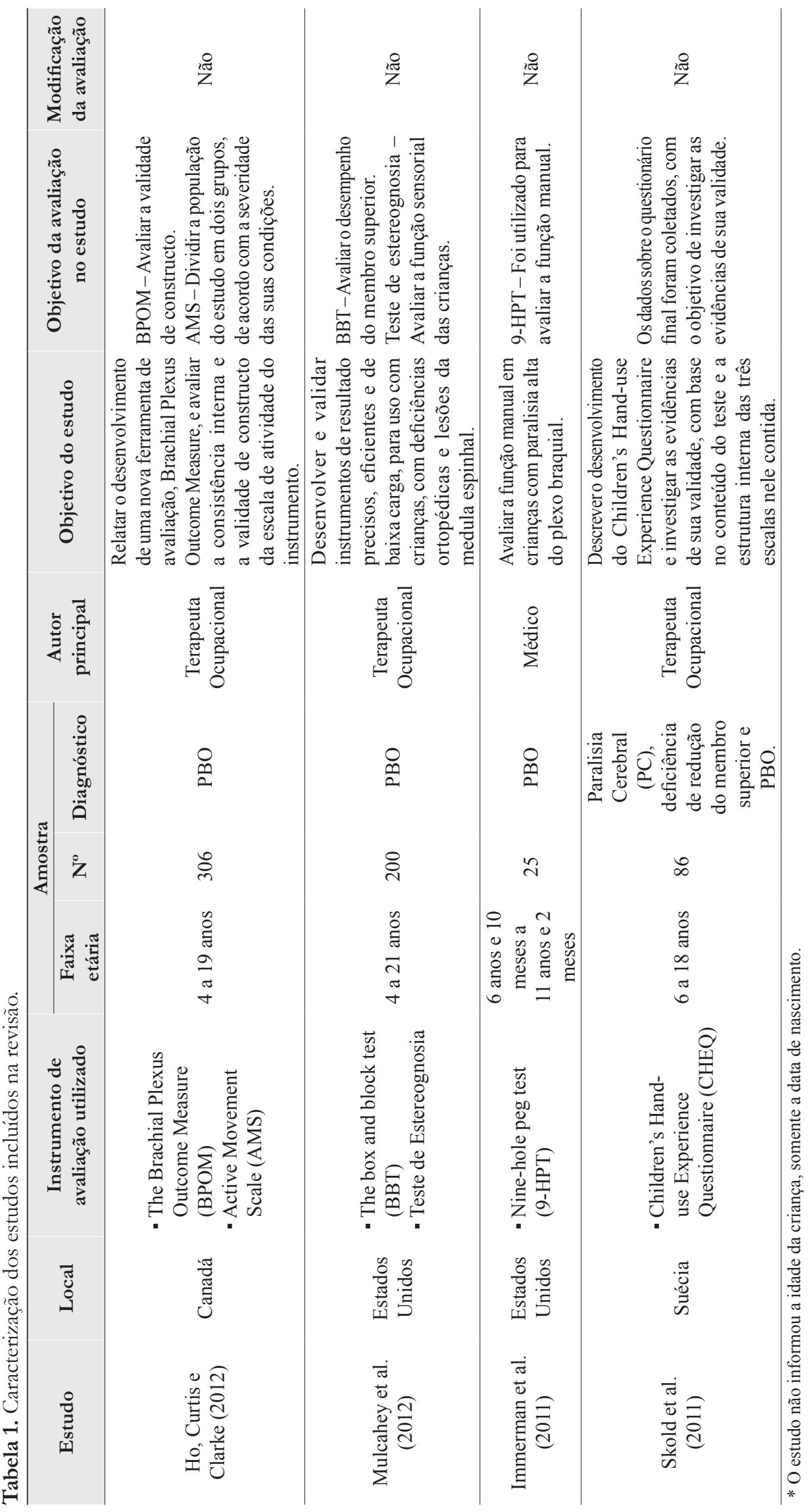




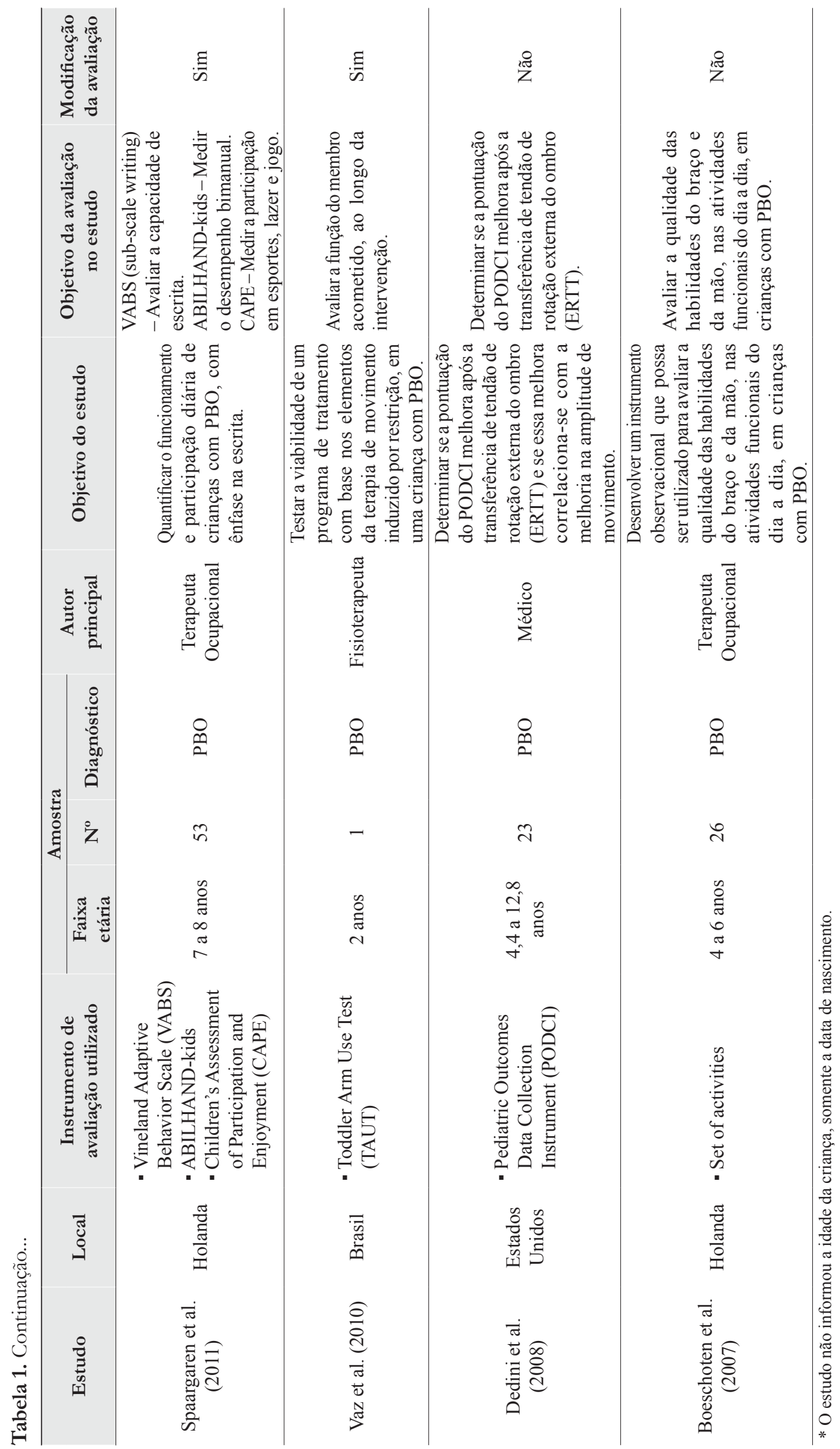




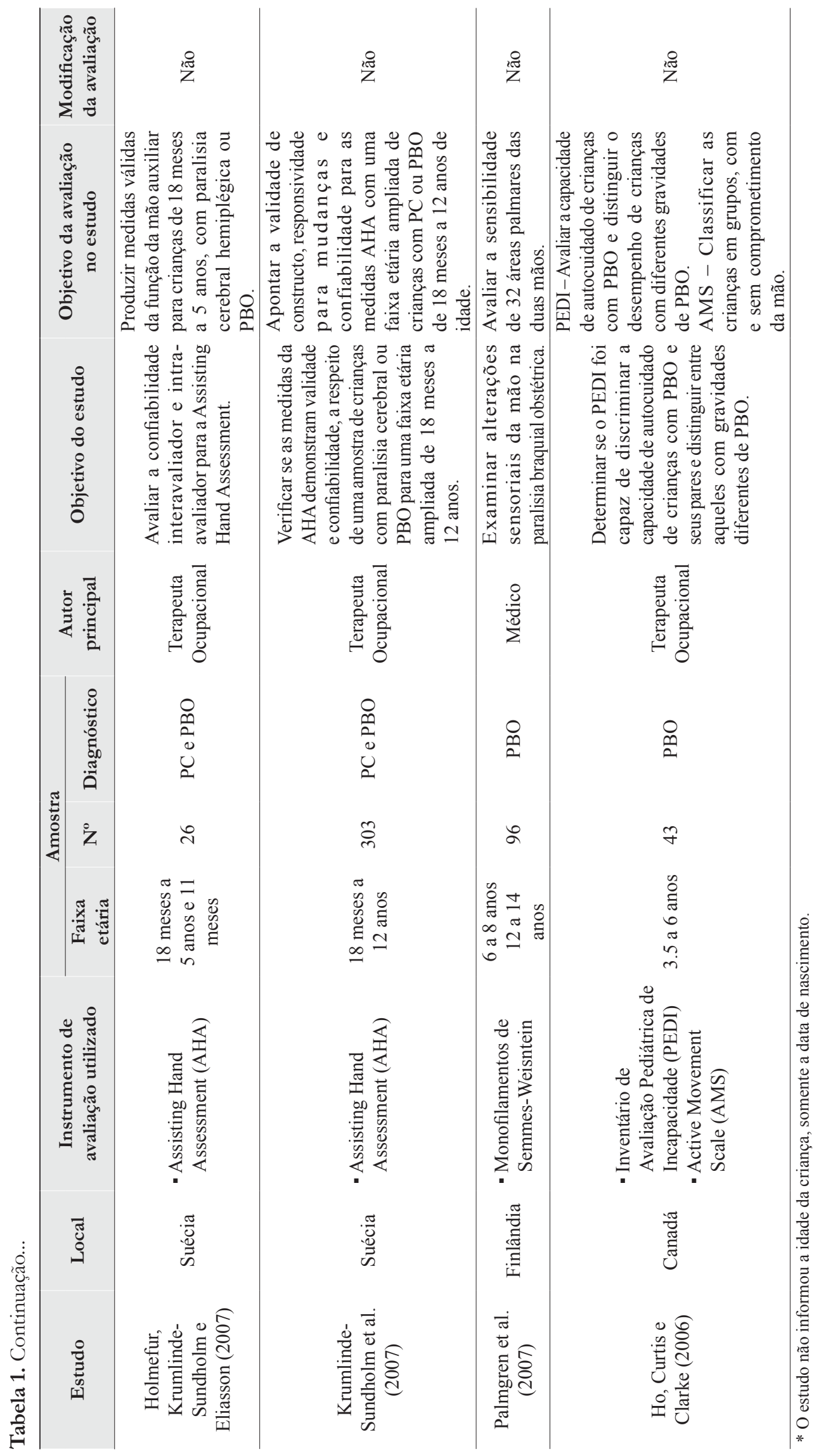




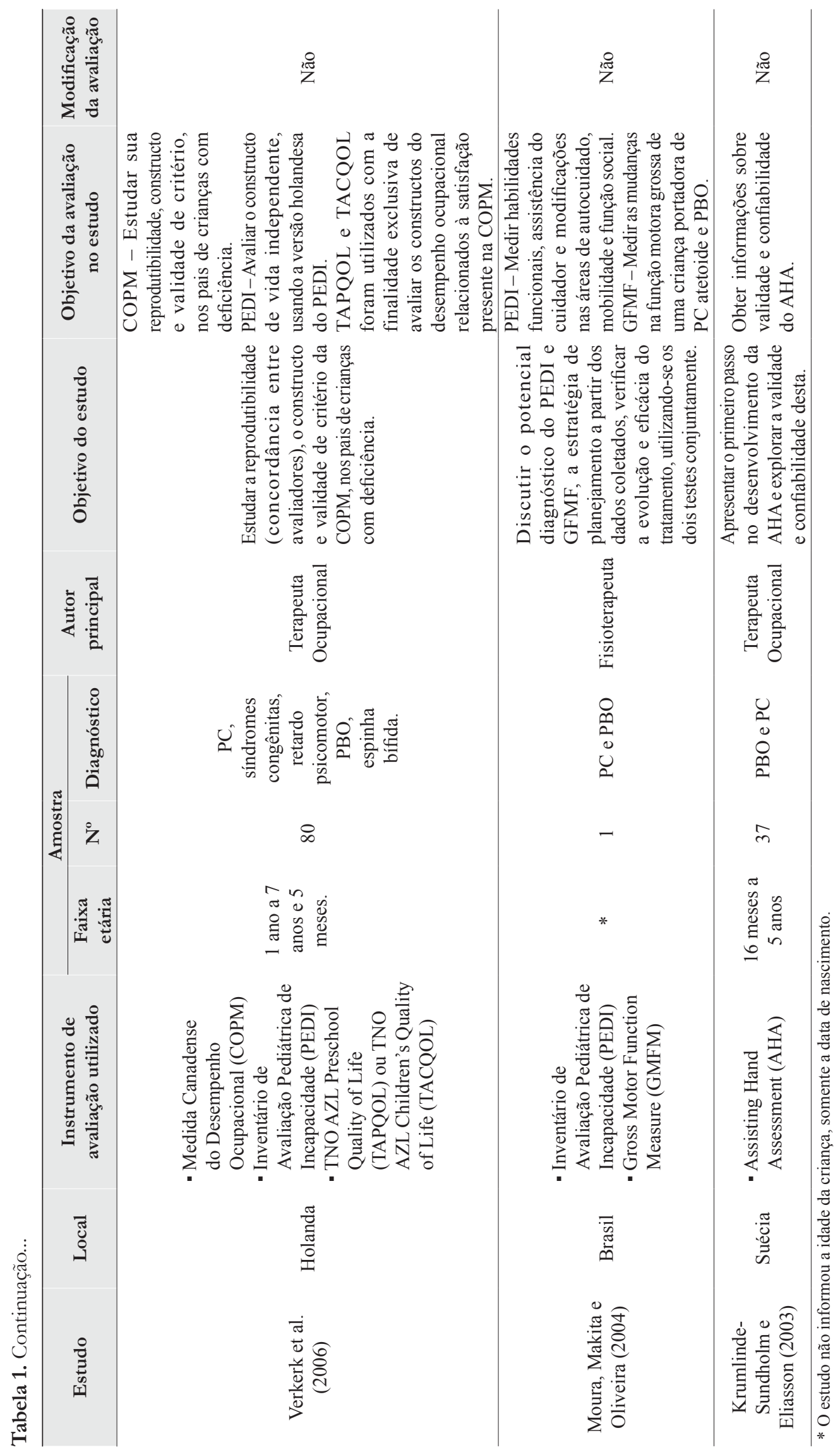




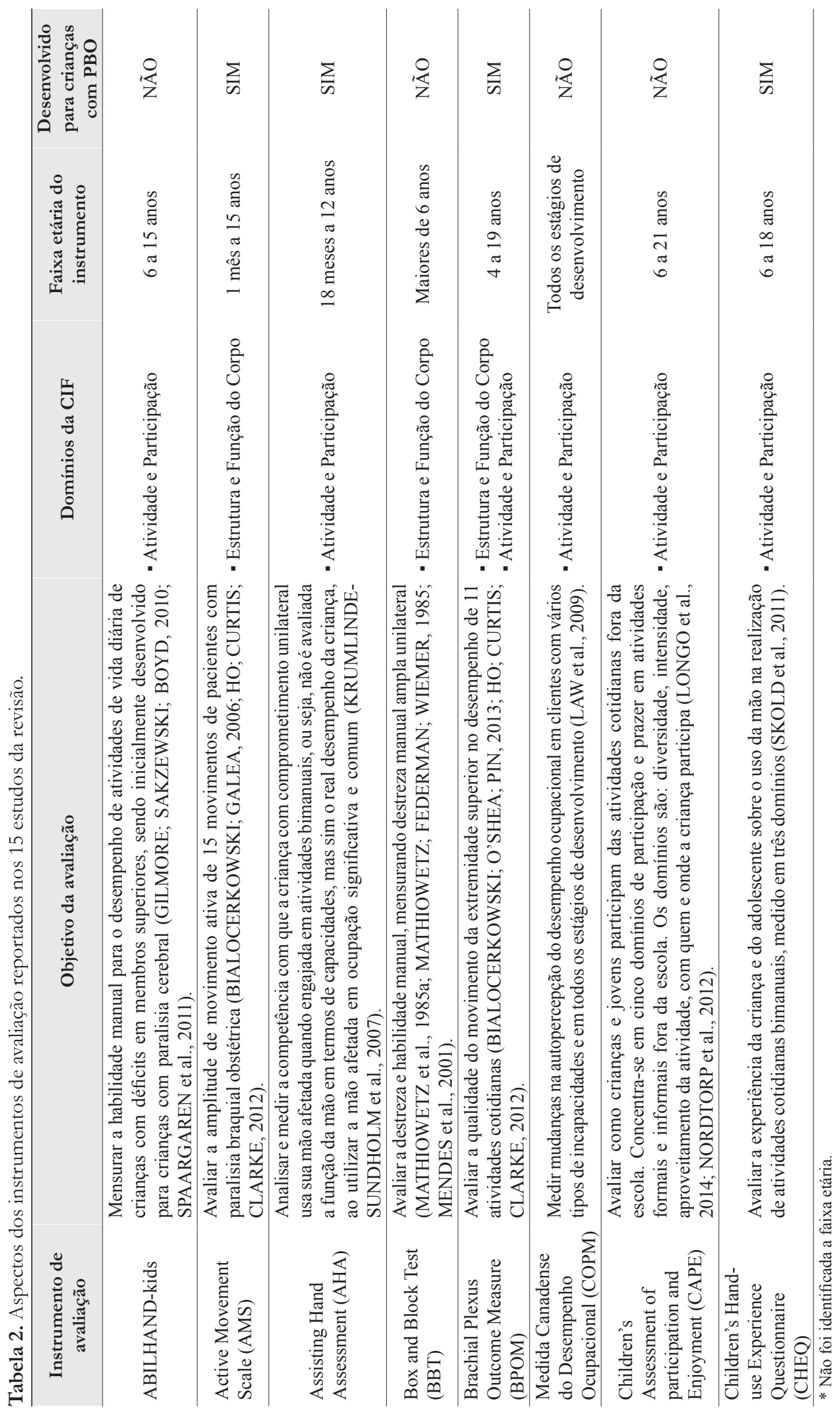




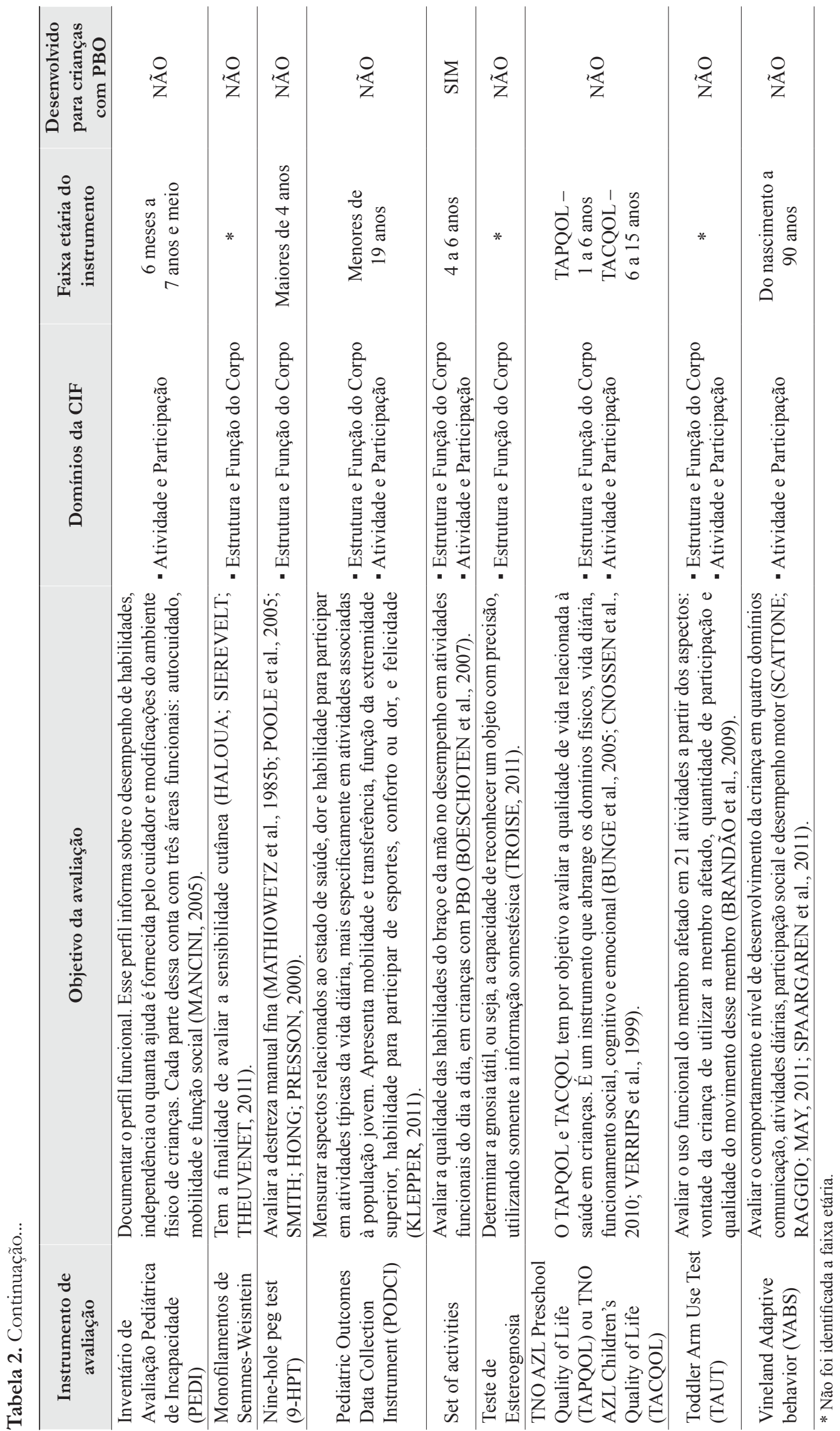


pediátrica, com base em uma abordagem centrada no cliente.

Dedini et al. (2008) pontuaram que o PODCI pode não ser sensível a mudanças na função do ombro. Porém, ressaltaram que em um estudo anterior (HUFFMAN et al., 2005) o instrumento foi capaz de mensurar diferenças entre o grupo controle e crianças com PBO.

Os objetivos dos instrumentos também se encontram descritos na Tabela 2. A partir da análise destes foi possível identificar que cinco estavam relacionados ao domínio da Classificação Internacional de Funcionalidade, Incapacidade e Saúde (CIF) de estrutura e função do corpo, sete à atividade e participação e cinco relacionados a ambos (Tabela 2).

As avaliaçóes utilizadas em maior número nos estudos foram a AHA (HOLMEFUR; KRUMLINDE-SUNDHOM; ELIASSON, 2007; KRUMLINDE-SUNDHOLM et al., 2007; KRUMLINDE-SUNDHOLM; ELIASSON, 2003) e o PEDI (HO; CURTIS; CLARKE, 2006; MOURA; MAKITA; OLIVEIRA, 2004; VERKERK et al., 2006). Em seguida, a avaliação mais usada foi a AMS, nos estudos de Ho, Curtis e Clarke (2006, 2012). As demais avaliaçóes foram aplicadas somente em um estudo (Tabela 1).

Em apenas dois estudos foram realizadas modificaçóes dos instrumentos de avaliação, (Tabela 1). No trabalho de Spaargaren et al. (2011), o subdomínio Escrita da VABS teve a remoção do item Escrever cartas comerciais; já em Vaz et al. (2010), o Toddler Arm Use Test (TAUT), em vez de ser utilizado com 21 tarefas funcionais e atividades lúdicas, foi utilizado com somente 15 .

Entretanto, Mulcahey et al. (2012) em seu estudo, apesar de não realizarem modificaçôes, sugeriram em seus resultados que estas deveriam ser feitas. Propuseram a adaptação do Teste de Estereognosia, reduzindo de doze para dois itens (moeda e botáo).

\section{Discussão}

A Suécia foi o país com maior número de publicações, com quatro artigos, o que pode ser atribuído pelo fato desse país ter uma incidência da doença considerada alta, apontada por Spaargaren et al. (2011) em 3,3 por 1000 nascidos vivos, uma vez que a incidência em países desenvolvidos é de 1,5 por nascidos vivos, de acordo com Vaz et al. (2010). Além disso, a Suécia foi o local de desenvolvimento de duas avaliaçóes específicas para a clientela de crianças com $\mathrm{PBO}$,
AHA e o CHEQ (KRUMLINDE-SUNDHOLM; ELIASSON, 2003; SKOLD et al., 2011).

A idade predominante das crianças dos estudos analisados foi de 4 a 7 anos, o que pode estar relacionado com a observação de Boeschoten et al. (2007) de que nessa faixa etária (4 a 6 anos) as limitaçóes oriundas da $\mathrm{PBO}$ se mostram mais óbvias, pois é quando o desempenho das crianças tem um aumento no número de atividades que necessitam envolvimento de ambas as mãos e braços.

Aproximadamente metade dos estudos ( $\mathrm{n}=7$ ) teve por objetivo buscar avaliaçóes que se adequassem à população de crianças com $\mathrm{PBO}$ (BOESCHOTEN et al., 2007; HO; CURTIS; CLARKE, 2006, 2012; KRUMLINDE-SUNDHOM; ELIASSON, 2003; MOURA; MAKITA; OLIVEIRA, 2004; MULCAHEY et al., 2012; SKOLD et al., 2011), e quatro destes tiveram como objetivo reportar o processo de desenvolvimento de alguns instrumentos específicos para tal clientela. Desses sete, somente um não teve como autor principal um terapeuta ocupacional. Essa informação sugere uma demanda dos terapeutas ocupacionais por instrumentos que possam avaliar crianças com $\mathrm{PBO}$, uma vez que os quatro trabalhos que relatam o desenvolvimento de instrumentos específicos tiveram como autor principal terapeutas ocupacionais (BOESCHOTEN et al., 2007; HO; CURTIS; CLARKE, 2012; KRUMLINDESUNDHOLM; ELIASSON, 2003; SKOLD et al., 2011).

Essa demanda pode ser justificada pelo fato de que é possível diagnosticar a gravidade das limitaçôes existentes, que dificultam o desempenho das atividades das crianças com $\mathrm{PBO}$, assim como planejar a intervenção e mensurar os seus efeitos através do processo de avaliação (BOESCHOTEN et al., 2007).

Quatro estudos tiveram por objetivo o desenvolvimento de avaliaçóes para crianças com $\mathrm{PBO}$, incluindo o CHEQ, a AHA, o BPOM e o Set of activities, em que os dois primeiros instrumentos se destinam a mensurar aspectos referentes ao domínio de Atividade e Participação da CIF, e os dois últimos, por sua vez, avaliam fatores relacionados a ambos os domínios Atividade e Participação e Estrutura e Função do Corpo (BOESCHOTEN et al., 2007; HO; CURTIS; CLARKE, 2012; KRUMLINDESUNDHOLM; ELIASSON, 2003; SKOLD et al., 2011) (Tabela 2).

Essa informação reitera a afirmação de $\mathrm{Ho}$, Curtis e Clarke (2012), de que é necessário o desenvolvimento de avaliaçóes que englobem aspectos relacionados ao domínio de Atividade e Participação, demonstrando que tais avaliaçóes, específicas para essa clientela, 
se adequam com o proposto pelo modelo de saúde preconizado pela CIF (FARIAS; BUCHALLA, 2005).

Em contrapartida, somente cinco instrumentos dos 17 identificados são específicos para crianças com $\mathrm{PBO}$, fato esse que pode estar associado à baixa incidência da doença (KRUMLINDE-SUNDHOLM; ELIASSON; FOISSBEIG, 1998). Corroborando com esse dado, o estudo de Foad, Mehlman e Ying (2008) demonstrou que a incidência de PBO nos hospitais dos Estados Unidos apresentou uma diminuição ao longo do tempo.

Desses cinco instrumentos específicos para $\mathrm{PBO}$, três foram desenvolvidos na Europa, o que é justificado pela alta incidência de $\mathrm{PBO}$ na regiáo e pelo aumento dessa incidência com o passar do tempo (SPAARGAREN et al., 2011). Esse aspecto epidemiológico pode estar relacionado à expressiva quantidade de artigos selecionados para o estudo $(\mathrm{N}=8)$ provenientes de países europeus (Tabela 1$)$.

Bialocerkowski, O'shea e Pin (2013) afirmam em seu estudo que as avaliaçóes utilizadas com crianças com $\mathrm{PBO}$, que apresentam evidências psicométricas mais robustas, incluem a AMS, a AHA, o PEDI e o PODCI. O fato da AHA, do PEDI e da AMS apresentarem boas propriedades psicométricas justifica a maior utilização destas, encontrada nessa revisão, uma vez que isso garante maior confiabilidade aos resultados.

A AHA foi uma das avaliações mais utilizadas nos estudos, e isso pode ser explicado pelo fato de ser um instrumento específico para crianças com PBO, assim como por ter sido desenvolvida há dez anos e ser um instrumento validado (KRUMLINDESUNDHOLM et al., 2007). Em contrapartida, a maior parte dos outros instrumentos específicos para PBO (CHEQ, Set of activities, BPOM e AMS) ainda estấo em processo de desenvolvimento e padronização, com exceção da AMS, que foi desenvolvida em 1995 (BIALOCERKOWSKI; GALEA, 2006; BOESCHOTEN et al., 2007; HO; CURTIS; CLARKE, 2012; SKOLD et al., 2011).

Os três estudos que utilizaram a AHA se propuseram a comprovar sua validade, confiabilidade e responsividade para a mudança, que são variáveis denominadas propriedades psicométricas. A variável validade é definida como a capacidade de um instrumento de medir com precisão um fenômeno, e a confiabilidade é a capacidade de medir um fenômeno fielmente. Já a responsividade para mudança é a capacidade do instrumento de constatar alteraçóes ao longo do tempo (BIALOCERKOWSKI; O'SHEA; PIN, 2013; PILATTI; PEDROSO; GUTIERREZ, 2010).
O artigo de Krumlinde-Sundholm et al. (2007) se propôs ainda a ampliar a faixa etária de aplicação da AHA de 18 meses a 5 anos para crianças de 18 meses a 12 anos, o que reitera a necessidade de instrumentos bem fundamentados para avaliar crianças com $\mathrm{PBO}$, pois uma vez comprovada sua validade e confiabilidade, foi pensado então em abranger mais crianças com essa avaliação.

No estudo de Silva et al. (2013), o autor pontuou que o PEDI se mostrou um importante instrumento para profissionais de saúde, em específico, terapeutas ocupacionais, uma vez que fornece descrição detalhada do desempenho funcional, permitindo a intervenção de forma estratégica nas dificuldades de cada criança. Além disso, Ho, Curtis e Clarke (2006) reportaram que a validade de constructo e confiabilidade do PEDI foi estabelecida em diversas populaçóes de crianças com comprometimento físico. O fato de ele ser amplamente utilizado com a população pediátrica pode justificar a sua utilizaçáo em três dos estudos (HO; CURTIS; CLARKE, 2006; MOURA; MAKITA; OLIVEIRA, 2004; VERKERK et al., 2006) incluídos nessa revisão.

A menção que Ho, Curtis e Clarke (2006) fizeram em seu trabalho sobre o PEDI náo ser capaz de discriminar a capacidade de autocuidado entre crianças com $\mathrm{PBO}$ e seus pares, não pode ser entendida como crítica à aplicabilidade do instrumento a essa população e sim como uma inadequação deste para estabelecer essa comparação, uma vez que o objetivo principal do PEDI é descrever o desempenho funcional da criança nas áreas de autocuidado, mobilidade e função social (MANCINI, 2005).

A AMS foi utilizada em dois estudos (HO; CURTIS; CLARKE, 2006, 2012), e isso também pode se referir às suas boas propriedades psicométricas, sendo confiável para aplicação em crianças (CURTIS et al., 2002). Nesses estudos, ela foi utilizada somente com o objetivo de classificar as crianças em dois grupos, de acordo com a severidade de suas condições: crianças com e sem comprometimento da mão. Em ambos, ela foi combinada com outra avaliação (BPOM e PEDI), o que pode indicar que sozinha não avalia amplamente a criança, pois está focada apenas em função do corpo, sendo também utilizado o PEDI e o BPOM para complementação de dados, uma vez que esses instrumentos avaliam domínios de Atividade e Participação.

Algumas avaliaçóes se propuseram a mensurar somente aspectos dos domínios de Estrutura e Função do Corpo da CIF, como o BBT, Teste de estereognosia, Monofilamentos de Semmes-Weisnten, 9-HPT e AMS. Dentre elas, os Monofilamentos de Semmes-Weisntein e o Teste de Estereognosia se 
destinam a avaliar aspectos sensoriais. $\mathrm{O}$ primeiro foi utilizado por Palmgren et al. (2007) com o objetivo de avaliar as alteraçóes sensoriais da mão na paralisia braquial obstétrica, e o segundo foi aplicado por Mulcahey et al. (2012) para avaliar a função sensorial das crianças.

$\mathrm{O}$ fato de terem sido identificados somente dois instrumentos com esse propósito pode ser devido à recuperação sensorial da máo ser geralmente boa na $\mathrm{PBO}$, especialmente em lesóes superiores do plexo, que são as lesôes mais comuns, e ainda pelo fato de a percepçáo fina da mão afetada estar raramente comprometida em crianças com lesão alta (PALMGREN et al., 2007; ZAFEIRIOU; PSYCHOGIOU, 2008). Ainda de acordo com Palmgren et al. (2007), a maioria das crianças recuperam a sensibilidade da máo próximo da normalidade na idade escolar, sendo assim, é possível considerar que náo exista muito interesse em avaliar esse aspecto, ou que náo seja muito relevante ter diversos instrumentos para mensurar tal aspecto, uma vez que existem instrumentos bem consolidados, com esse propósito como os citados anteriormente.

A Avaliação da Função Motora Grossa (GMFM), utilizada no estudo de Moura, Makita e Oliveira (2004), não foi incluída nos resultados por não ser considerada relevante para esta revisão, uma vez que é específica para mensurar mudanças na função motora grossa de crianças com Paralisia Cerebral (PC) e foi utilizada em razão de a criança estudada ter diagnóstico de $\mathrm{PC}$ e $\mathrm{PBO}$ concomitantemente.

Alguns autores fizeram modificaçôes nos instrumentos, especificamente no VABS e no TAUT. Vaz et al. (2010) realizaram a redução para 15 tarefas funcionais e atividades lúdicas do TAUT, por considerarem que dentre as 21 tarefas propostas pelo instrumento, estas 15 se adequavam a crianças com 2 anos de idade. Em relação à VABS, no trabalho de Spaargaren et al. (2011) a remoção do item Escrever cartas comerciais, do subdomínio referente à escrita, foi realizada por esse item ser considerado inadequado para crianças de 7 a 8 anos de idade.

Desse modo, é possível inferir que as modificaçóes realizadas não tiveram o objetivo de adequar os instrumentos supracitados à populaçáo de crianças com PBO, e sim à faixa etária da amostra estudada, o que sugere que as avaliaçóes identificadas em seu formato original são passíveis de aplicação a crianças com PBO.

Mulcahey et al. (2012) não apresentaram modificação do instrumento, porém com base nos seus resultados sugeriram que pode ser viável a adaptação do Teste de Estereognosia com a redução de doze para dois itens (moeda e botáo), sem haver comprometimento das informações significativas sobre a função sensorial da criança. Ainda de acordo com os autores, embora possa parecer insignificante testar dois itens em vez de doze, essa modificação cria a possibilidade de reduzir o tempo necessário para a realização do teste, diminuindo, portanto, a carga sobre a criança e tornando a avaliaçáo menos desgastante.

A diversidade de instrumentos identificados com diferentes objetivos deixa clara a heterogeneidade dos comprometimentos da paralisia braquial obstétrica, pois como apontado por Bialocerkowski e Galea (2006) e Krumlinde-Sundholm, Eliasson e Foissbeig (1998), a PBO pode gerar diversos comprometimentos, incapacidades e dificuldades funcionais associadas com o uso do membro superior.

\section{Conclusão}

A partir dos resultados dessa revisão, é possível constatar que a produção da Terapia Ocupacional em relação a crianças com Paralisia Braquial Obstétrica ainda é pequena e que poucos instrumentos de avaliação são específicos para esta clientela.

Entretanto, foram identificadas diferentes avaliaçôes que podem ser empregadas pelo terapeuta ocupacional com crianças com PBO, contemplando diferentes objetivos e abrangendo em sua maioria aspectos referentes aos domínios da CIF de Atividade e Participação, indicando a preocupação em adequar o processo de avaliaçáo ao paradigma atual da compreensão de saúde que utiliza o indicador da funcionalidade como parâmetro.

Identificou-se o uso de instrumentos não específicos para crianças com $\mathrm{PBO}$, porém passíveis de aplicação com essa clientela, não sendo verificada necessidade de modificação de tais instrumentos para viabilizar esse uso, o que facilita o emprego destes na prática clínica, contribuindo para avaliar e registrar os efeitos da intervenção.

Foi observado um aumento no interesse de terapeutas ocupacionais em desenvolver instrumentos de avaliação específicos para a clientela de crianças com $\mathrm{PBO}$, que se caracterizam pelo foco no desempenho funcional e ocupacional, demonstrando a preocupação destes profissionais em produzir conhecimentos e evidências que fundamentem a prática clínica.

Cada instrumento empregado nos estudos apresenta particularidades, vantagens e desvantagens, assim sendo, cabe ao terapeuta ocupacional, no momento de escolher a avaliação, levar em consideração qual é a mais adequada aos seus objetivos e à idade 
da criança, tanto para a utilização em pesquisas, triagem clínica ou na verificação da eficiência da intervenção. Além disso, devem-se considerar no processo de seleção de instrumentos de avaliação as propriedades psicométricas destes, pois bons valores de confiabilidade e validade são fundamentais na eficácia do teste.

Diante do exposto, cabe reiterar a necessidade contínua do investimento de terapeutas ocupacionais no desenvolvimento de instrumentos de avaliação para crianças com Paralisia Braquial Obstétrica que possuam boas propriedades psicométricas. Espera-se ainda que a presente revisão possa contribuir no processo de escolha de avaliaçóes adequadas que fundamentem e comprovem a eficácia da intervenção do terapeuta ocupacional junto a essa clientela.

\section{Referências}

BIALOCERKOWSKI, A.; GALEA, M. Comparison of visual and objective quantification of elbow and shoulder movement in children with obstetric brachial plexus palsy. Journal of Brachial Plexus and Peripheral Nerve Injury, London, v. 1, n. 5, p. 1-6, 2006. PMid:17147776.

BIALOCERKOWSKI, A.; GELDING, B. Lack of evidence of the effectiveness of primary brachial plexus surgery for infants (under the age of two years) diagnosed with obstetric brachial plexus palsy. International Journal of Evidence Based Healthcare, Carlton South, v. 4, n. 4, p. 264-287, 2006. PMid:21631772.

BIALOCERKOWSKI, A.; O'SHEA, K.; PIN, T. W. Psychometric properties of outcome measures for children and adolescents with brachial plexus birth palsy: a systematic review. Developmental Medicine and Child Neurology, Oxford, v. 55, n. 12, p. 10751088, 2013. http://dx.doi.org/10.1111/dmcn.12194. PMid:23808952.

BOESCHOTEN, K. H. et al. Development of a set of activities to evaluate the arm and hand function in children with obstetric brachial plexus lesion. Clinical Rehabilitation, London, v. 21, n. 2, p. 163-170, 2007. http://dx.doi.org/10.1177/0269215506071253. PMid:17264110.

BRANDĀO, M. B. et al. Effects of constraint-induced movement therapy in children with hemiplegia: a single case experimental study. Revista Brasileira de Fisioterapia, São Carlos, v. 13, n. 6, p. 527-534, 2009. http://dx.doi. org/10.1590/S1413-35552009005000064.

BRAYMAN, S. J. et al. Standards of practice for occupational therapy. American Journal of Occupational Therapy, Bethesda, v. 59, n. 6, p. 663-665, 2005. http://dx.doi. org/10.5014/ajot.59.6.663. PMid:16363189.

BUNGE, E. M. et al. Reliability and validity of health status measurement by the TAPQOL. Archives of Disease in Childhood, London, v. 90, n. 4, p. 351-358,
2005. http://dx.doi.org/10.1136/adc.2003.048645. PMid:15781921.

CHAVES, G. F. S. et al. Escalas de avaliação para Terapia. Revista de Terapia Ocupacional da Universidade de São Paulo, São Paulo, v. 21, n. 3, p. 240-246, 2010.

CNOSSEN, M. H. et al. Paediatric arterial ischaemic stroke: functional outcome and risk factors. Developmental Medicine e Child Neurology, Oxford, v. 52, n. 4, p. 394-399, 2010. http://dx.doi.org/10.1111/j.14698749.2009.03580.x. PMid:20089051.

CURTIS, C. et al. The Active Movement Scale: an evaluative tool for infants with obstetrical brachial plexus palsy. Journal Hand Surgery, Edinburgh, v. 27, n. 3, p. 470-478, 2002. http://dx.doi.org/10.1053/ jhsu.2002.32965. PMid:12015722.

DEDINI, R. D. et al. Comparison of pediatric outcomes data collection instrument scores and range of motion before and after shoulder tendon transfers for children with brachial plexus birth palsy. Journal of Pediatric Orthopaedics, New York, v. 28, n. 2, p. 259-264, 2008. http://dx.doi.org/10.1097/BPO.0b013e3181652135. PMid:18388726.

FARIAS, N.; BUCHALLA, C. M. A. A Classificaçāo Internacional de Funcionalidade, Incapacidade e saúde da Organização mundial da saúde: conceitos, usos e perspectivas. Revista Brasileira de Epidemiologia, São Paulo, v. 8, n. 2, p. 187-193, 2005. http://dx.doi.org/10.1590/ S1415-790X2005000200011.

FOAD, S. L.; MEHLMAN, C. T.; YING, J. The epidemiology of neonatal brachial plexus Palsy in the United States. Journal of Bone and Joint Surgery American, Boston, v. 90, n. 6, p. 1258-1264, 2008. http://dx.doi. org/10.2106/JBJS.G.00853.

GHIZONI, M. F. et al. Paralisia obstétrica de plexo braquial: revisão da literatura. Arquivos Catarinenses de Medicina, Florianópolis, v. 39, n. 4, p. 95-101, 2010.

GILMORE, R.; SAKZEWSKI, L.; BOYD, R. Upper limb activity measures for 5- to 16-year-old children with congenital hemiplegia: a systematic review. Developmental Medicine e Child Neurology, Oxford, v. 52, n. 1, p. 14-21, 2010. http://dx.doi.org/10.1111/j.14698749.2009.03369.x. PMid:19811513.

HALOUA, M. H.; SIEREVELT, I.; THEUVENET, W. J. Semmes-weinstein monofilaments: influence of temperature, humidity, and age. Journal of Hand Surgery, Oxford, v. 36, n. 7, p. 1191-1196, 2011. http://dx.doi. org/10.1016/j.jhsa.2011.04.009. PMid:21712138.

HO, E. S.; CURTIS, C. G.; CLARKE, H. M. Pediatric Evaluation of Disability Inventory: its application to children with obstetric brachial plexus palsy. Journal of Hand Surgery, New York, v. 31, n. 2, p. 197-202, 2006. http://dx.doi.org/10.1016/j.jhsa.2005.10.007. PMid:16473678.

HO, E. S.; CURTIS, C. G.; CLARKE, H. M. The brachial plexus outcome measure: development, in- 
ternal consistency, and construct validity. Journal of Hand Therapy, Philadelphia, v. 25, n. 4, p. 406-416, 2012. http://dx.doi.org/10.1016/j.jht.2012.05.002. PMid:22818900.

HOEKSMA, A. F; WOLF, H.; OEI, S. L. Obstetrical brachial plexus injuries: incidence, natural course and shoulder contracture. Clinical Rehabilitation, London, v. 14, n. 5, p. 523-526, 2000. http://dx.doi. org/10.1191/0269215500cr341oa. PMid:11043878.

HOLMEFUR, M.; KRUMLINDE-SUNDHOM, L.; ELIASSON, A. Interrater and intrarater reliability of the Assisting Hand Assessment. American Journal of Occupational Therapy, Bethesda, v. 61, n. 1, p. 79-84, 2007. http://dx.doi.org/10.5014/ajot.61.1.79. PMid:17302108.

HUFFMAN, G. R. et al. Assessment of children with brachial plexus birth palsy using the Pediatric Outcomes Data Collection Instrument. Journal of Pediatric Orthopaedics, New York, v. 25, n. 3, p. 400-404, 2005. http:// dx.doi.org/10.1097/01.bpo.0000151055.62356.1b. PMid:15832163.

IMMERMAN, I. et al. Hand function in children with an upper brachial plexus injury: results of the nine-hole peg test. Developmental Medicine e Child Neurology, Oxford, v. 54, n. 2, p. 166-169, 2011. http:// dx.doi.org/10.1111/j.1469-8749.2011.04120.x. PMid:22129161.

KLEPPER, S. E. Measures of pediatric function: Child Health Assessment Questionnaire (C-HAQ), Juvenile Arthritis Functional Assessment Scale (JAFAS), Pediatric Outcomes Data Collection Instrument (PODCI), and Activities Scale for Kids (ASK). Arthritis Care \& Research, Atlanta, v. 63, n. 11, p. 371-382, 2011. http:// dx.doi.org/10.1002/acr.20635. PMid:22588758.

KRUMLINDE-SUNDHOLM, L. et al. The Assisting Hand Assessment: current evidence of validity, reliability, and responsiveness to change. Developmental Medicine e Child Neurology, Oxford, v. 49, n. 4, p. 259-264, 2007. http://dx.doi.org/10.1111/j.1469-8749.2007.00259.x. PMid:17376135.

KRUMLINDE-SUNDHOLM, L.; ELIASSON, A. Development of the Assisting Hand Assessment: a rasch-built measure intended for children with unilateral upper limb impairments. Scandinavian Journal of Occupational Therapy, Oslo, v. 10, n. 1, p. 16-26, 2003. http://dx.doi.org/10.1080/11038120310004529.

KRUMLINDE-SUNDHOLM, L.; ELIASSON, A.; FOISSBEIG, H. Obstetric brachial plexus injuries: assessment protocol and functional outcome at age 5 years. Developmental Medicine e Child Neurology, Oxford, v. 40, n. 1, p. 4-11, 1998. http:// dx.doi.org/10.1111/j.1469-8749.1998.tb15350.x. PMid:9459211.

LAW, M. et al. Medida canadense de desempenho ocupacional (COPM). Belo Horizonte: Editora UFMG, 2009.
LONGO, E. et al. Cross-cultural validation of the Children's Assessment of Participation and Enjoyment (CAPE) in Spain. Child: Care Health and Development, Oxford, v. 40, n. 2, p. 231-241, 2014. PMid:23210505.

MANCINI, M. C. Inventário de Avaliação Pediátrica de Incapacidade (PEDI): manual da versão brasileira adaptada. Belo Horizonte: Editora UFMG, 2005.

MATHIOWETZ, V. G. et al. Adult norms for the box and block tests of manual dexterity. American Journal of Occupational Therapy, Bethesda, v. 39, n. 6, p. 386391, 1985a. http://dx.doi.org/10.5014/ajot.39.6.386. PMid:3160243.

MATHIOWETZ, V. G. et al. Adult norms for the Nine-Hole Peg Test of finger dexterity. Occupational Therapy Journal of Research, Thorofare, v. 5, n. 1, p. 24-38, $1985 \mathrm{~b}$

MATHIOWETZ, V. G.; FEDERMAN, S.; WIEMER, D. Box and block test of manual dexterity: norms for 6-19 year olds. Canadian Journal of Occupational Therapy, Toronto, v. 52, n. 5, p. 241-245, 1985. http://dx.doi. org/10.1177/000841748505200505.

MENDES, M. F. et al. Teste de destreza manual da caixa e blocos em indivíduos normais e em pacientes com esclerose múltipla. Arquivos de Neuropsiquiatria, São Paulo, v. 59, n. 4, p. 889-894, 2001. http://dx.doi. org/10.1590/S0004-282X2001000600010.

MOURA, E. W.; MAKITA, L. M.; OLIVEIRA, M. C. Utilização do PEDI e GMFM no planejamento de tratamento de criança com paralisia cerebral do tipo atetóide e paralisia braquial obstétrica. Temas sobre Desenvolvimento, São Paulo, v. 13, n. 73, p. 41-46, 2004.

MULCAHEY, M. J. et al. Evaluation of the box and blocks test, stereognosis and item banks of activity and upper extremity function in youths with brachial plexus birth palsy. Journal of Pediatric Orthopaedics, New York, v. 32, n. 2, p. 114-122, 2012. http://dx.doi.org/10.1097/ BPO.0b013e3182595423. PMid:22890449.

NORDTORP, H. L. et al. Reliability of the Norwegian Version of the Children's Assessment of Participation and Enjoyment (CAPE) and Preferences for Activities of Children (PAC). Physical \& Occupational Therapy in Pediatrics, New York, v. 33, n. 2, p. 199-212, 2012. http://dx.doi.org/10.3109/01942638.2012.739269. PMid:23157665.

PALMGREN, T. et al. Sensory evaluation of the hands in children with brachial plexus birth injury. Developmental Medicine e Child Neurology, Oxford, v. 49, n. 8, p. 582-586, 2007. http://dx.doi.org/10.1111/j.14698749.2007.00582.x. PMid:17635202.

PILATTI, L. A.; PEDROSO, B.; GUTIERREZ, G. L. Propriedades psicométricas de instrumentos de avaliação: Um debate necessário. Revista Brasileira de Ensino de Ciência e Tecnologia, Ponta Grossa, v. 3, n. 1, p. 81-91, 2010 . 
POOLE, J. L. et al. Measuring dexterity in children using the Nine-hole Peg Test. Journal of Hand Therapy, Philadelphia, v. 18, n. 3, p. 348-351, 2005. http://dx.doi. org/10.1197/j.jht.2005.04.003. PMid:16059856.

ROCHA, S. R.; DORNELAS, L. F.; MAGALHĀES, L. C. Instrumentos utilizados para avaliação do desenvolvimento de recém-nascidos pré-termo no Brasil: revisão da literatura. Cadernos de Terapia Ocupacional da UFSCar, São Carlos, v. 21, n. 1, p. 109-117, 2013. http://dx.doi. org/10.4322/cto.2013.015.

SCATTONE, D.; RAGGIO, D. J.; MAY, W. Comparison of the vineland adaptive behavior scales, second edition, and the bayley scales of infant and toddler development, third edition. Psychological Reports, Montana, v. 109, n. 2, p. 626-634, 2011. http:// dx.doi.org/10.2466/03.10.PR0.109.5.626-634. PMid:22238860.

SILVA, S. et al. Paralisia do plexo braquial no recém-nascido: experiência de dez anos numa maternidade de apoio perinatal diferenciado. Acta Pediátrica Portuguesa, Lisboa, v. 41, n. 3, p. 127-130, 2010.

SILVA, V. F. et al. Análise do desempenho de autocuidado em crianças com Síndrome de Down. Cadernos de Terapia Ocupacional da UFSCar, São Carlos, v. 21, n. 1, p. 83-90, 2013. http://dx.doi.org/10.4322/cto.2013.012.

SIQUEIRA, R. Lesōes nervosas periféricas: uma revisão. Revista de Neurociência, São Paulo, v. 15, n. 3, p. 226233, 2007.

SKÖLD, A. et al. Development and evidence of validity for the Children's Hand-use Experience Questionnaire (CHEQ). Developmental Medicine e Child Neurology, Oxford, v. 53, n. 5, p. 436-442, 2011. http:// dx.doi.org/10.1111/j.1469-8749.2010.03896.x. PMid:21413973.

SMITH, Y. A.; HONG, E.; PRESSON, C. Normative and validation studies of the nine-hole peg test with children. Perceptual and Motor Skills, Missoula, v. 90, n. 3, p. $823-843,2000$. http://dx.doi.org/10.2466/ pms.2000.90.3.823. PMid:10883762.

SPAARGAREN, E. et al. Aspects of activities and participation of 7-8 year-old children with an obstetric brachial plexus injury. European Journal of Paediatric Neurology, London, v. 15, n. 4, p. 345-352, 2011. http://dx.doi. org/10.1016/j.ejpn.2011.03.008. PMid:21511503.

TROISE, D. C. Avaliação da somestesia e coordenação motora em crianças com distrofia muscular de Duchenne. 2011. 48 f. Dissertaçáo (Mestrado em Ciência da Reabilitação) - Universidade de São Paulo, São Paulo, 2011.

UNSWORTH, C. Measuring the outcome of occupational therapy: tools and resources. Australian $\mathrm{Oc}$ cupational Therapy Journal, Melbourne, v. 47, n. 4, p. 147-158, 2000. http://dx.doi.org/10.1046/j.14401630.2000.00239.x.

VAZ, D. V. et al. Clinical changes during an intervention based on constraint-induced movement therapy principles on use of the affected arm of a child with obstetric brachial plexus injury: a case report. Occupational Therapy International, London, v. 17, n. 4, p. 159-167, 2010. http://dx.doi.org/10.1002/oti.295. PMid:20645277.

VERKERK, G. J. Q. et al. The reproducibility and validity of the Canadian Occupational Performance Measure in parents of children with disabilities. Clinical Rehabilitation, London, v. 20, n. 11, p. 980-988, 2006. http://dx.doi.org/10.1177/0269215506070703. PMid:17065541.

VERRIPS, E. G. H. et al. Measuring health-related quality of life in a child population. European Journal of Public Health, Stockholm, v. 9, n. 3, p. 188-193, 1999. http://dx.doi.org/10.1093/eurpub/9.3.188.

ZAFEIRIOU, D. I.; PSYCHOGIOU, K. Obstetrical brachial plexus palsy. Pediatric Neurology, Chippewa Falls, v. 38, n. 4, p. 235-242, 2008. http:// dx.doi.org/10.1016/j.pediatrneurol.2007.09.013. PMid: 18358400.

\section{Contribuição dos Autores}

Thaianny Taís Dantas de Brito realizou a revisão bibliográfica e a elaboração do artigo. Carolinne Linhares Pinheiro colaborou na revisão bibliográfica, elaboração do artigo, realizou a revisão do texto e foi a responsável pela orientaçáo do trabalho. Ambas autoras aprovaram a versão final do texto. 